\title{
The Crossed Product of a UHF algebra by a Shift
}

by

\author{
Ola Bratteli, ${ }^{1)}$ Akitaka Kishimoto, ${ }^{2)}$ Mikael Rørdam, ${ }^{3)}$ Erling Størmer ${ }^{1)}$
}

\begin{abstract}
We prove that the crossed product of the CAR algebra $M_{2 \infty}$ by the shift is an inductive limit of homogeneous algebras over the circle with fibres full matrix algebras. As a consequence the crossed product has real rank zero, and $\mathcal{O}_{2} \cong \mathcal{O}_{2} \otimes M_{2 \infty}$ where $\mathcal{O}_{2}$ is the Cuntz algebra of order 2.
\end{abstract}

\section{Introduction}

Let $M_{2 \infty}$ be the CAR algebra, i.e. the UHF algebra of Glimm type $2^{\infty}$, [BR2], [Gli]. Then $M_{2 \infty}=\bigotimes_{-\infty}^{\infty} M_{2}$. It is well-known that if $\beta$ is an automorphism of $M_{2 \infty}$ of product type, then the crossed product $M_{2^{\infty}} \times_{\beta} \mathrm{Z}$ is an inductive limit of algebras of the form $M_{2^{n}} \otimes C(\mathrm{~T})$, where $\mathbf{T}$ is the circle, [Bra2, Theorem 2.1]. In this paper we will prove a similar result in the much more nontrivial situation that $\alpha$ is an automorphism of $M_{2 \infty}$ with strong ergodicity properties. Let $\alpha$ be the (Bernoulli) shift of $M_{2^{\infty}}$ obtained by translating each tensor factor by one to the right [BR1, Example 4.3.26]. The crossed product $\mathcal{B}=M_{2^{\infty}} \times{ }_{\alpha} \mathrm{Z}$ is then a simple unital $C^{*}$-algebra, [Kis].

The algebra $\mathcal{B}$ has a canonical trace state obtained by extending the unique trace state $\tau$ on $M_{2 \infty}$ to $\mathcal{B}$ by

$$
\tau\left(\sum_{n} a_{n} u^{n}\right)=\tau\left(a_{0}\right)
$$

where $a_{n} \in M_{2^{\infty}}$ and $u$ is the canonical unitary in $\mathcal{B}$ implementing $\alpha$. This is the only trace state on $\mathcal{B}$ by the following reasoning: By [Bed] it suffices to show that the extension of the shift to the weak closure of $M_{2 \infty}$ in the cyclic trace representation $\left(\pi_{\tau}, \mathcal{H}_{\tau}, \Omega_{\tau}\right)$ is outer. But

$$
\lim _{n \rightarrow \infty} \tau\left(a \alpha^{n}(b)\right)=\tau(a) \tau(b)
$$

\footnotetext{
1) Department of Mathematics, University of Oslo, P.O.Box 1053 Blindern, N-0316 Oslo 3, Norway

2) Department of Mathematics, Hokkaido University, Sapporo, 060 Japan

3) Department of Mathematics and Computer Science, Odense University, DK-5230 Odense M, Denmark
} 
for all $a, b \in M_{2 \infty}$ and hence the projection $E_{\omega}$ onto the $u_{\tau}(\alpha)$-invaviant vectors in $\mathcal{H}_{\tau}$ is one-dimensional, where $u_{\tau}(\alpha)$ is the canonical unitary operator implementing $\alpha$ in the trace representation, [BR1, Theorem 4.3.22]. It follows that the extension of $\alpha$ to $\pi_{\tau}(\mathcal{A})^{\prime \prime}$ is ergodic, [BR1, Theorem 4.3.20], and thus this extension is outer.

We conclude that $\mathcal{B}$ has a unique trace state.

The main result of this paper is

Theorem 1.1 There is an increasing sequence $\mathcal{B}_{n}$ of $C^{*}$-subalgebras of $\mathcal{B}$ such that $\cup_{n} \mathcal{B}_{n}$ is dense in $\mathcal{B}$, and each $\mathcal{B}_{n}$ has the form

$$
\mathcal{B}_{n} \cong \bigoplus_{k=1}^{m_{n}} M_{[n, k]} \otimes C(\mathrm{~T})
$$

where $[n, k] \in \mathbf{N}$, and $m_{n}$ is finite. In particular $\mathcal{B}$ has real rank zero.

Recall from $[\mathrm{BP}]$ that $\mathcal{B}$ is said to have real rank zero if for any $x=x^{*} \in \mathcal{B}$ and any $\varepsilon>0$ there exists a $y=y^{*} \in \mathcal{B}$ such that $y$ has finite spectrum and $\|x-y\|<\varepsilon$. As soon as we have established that $\mathcal{B}$ is an inductive limit of finite direct sum of circle algebras, it follows from the uniqueness of the trace state that the projections in $\mathcal{B}$ trivially separate the trace states, and hence $\mathcal{B}$ has real rank zero by [BBEK, Theorem 1.3] or [BDR, Theorem 2]. Thus the last statement in Theorem 1.1 is a consequence of the first. We will prove the first statement in Section 5.

As corollaries of Proposition 4.1, established in the course of the proof, we also deduce

Corollary 1.2: $\quad \mathcal{B} \cong \mathcal{B} \otimes M_{2^{\infty}}$

Corollary 1.3: $\mathcal{O}_{2} \cong \mathcal{O}_{2} \otimes M_{2 \infty}$, where $\mathcal{O}_{2}$ is the Cuntz algebra of order 2, [Cun].

Remark 1.4 : Once Theorem 1.1 is established, one may use Elliott's classification in [Ell], [Su] to say more about the increasing sequence $\mathcal{B}_{n}$. One may for example take

$$
\mathcal{B}_{n} \cong M_{4^{n}} \otimes C(\mathrm{~T})
$$

and the embedding $\mathcal{B}_{n} \hookrightarrow \mathcal{B}_{n+1}$ to be 2 copies of the twice around embedding, [Bla2]. This is seen as follows:

We first compute the $\mathrm{K}$-theory of $\mathcal{B}$. By the Pimsner-Voiculescu exact 6-term sequence, the $\mathrm{K}$-groups of $\mathcal{B}$ are given by

$$
K_{0}(\mathcal{B}) \cong \mathbf{Z}\left[\frac{1}{2}\right] \cong K_{1}(\mathcal{B})
$$


[Bla 1, 10.2.1]. Representatives in the $K_{0}$-and $K_{1}$-classes corresponding to a dyadic rational can be described explicitely as follows:

If $p$ is a projection in the CAR algebra $\mathcal{A}$ with trace $\tau(p) \in \mathbf{Z}\left[\frac{1}{2}\right] \cap[0,1]$, then $p$ is contained in the $K_{0}$ class $\tau(p)$ in $K_{0}(\mathcal{B})$. Since $1-p$ and $1-u p u^{*}$ are equivalent in the CAR algebra, there exists a partial isometry $v \in \mathcal{A}$ which implements this equivalence. Now $u p+v$ is an element in the $K_{1}$-class $\tau(p)$. For dyadic rationals outside $[0,1]$ one may construct representatives in the $K$-classes by using matrix algebras over $\mathcal{A}$ or by considereing unitaries of the form $u^{n} p+v_{n}$, where $n \in \mathbf{Z}$.

Next note that $K_{0}(\mathcal{B})$ as an ordered group with order unit is the dyadic rationals with positive cone equal to the non-negative dyadic numbers and order unit equal to 1 . This is because the positive cone in $\mathcal{A}$ is contained in the positive cone in $\mathcal{B}$. Assume that $x$ belongs to the positive cone in $K_{0}(\mathcal{B})$, and that $x \neq 0$. As $\mathcal{B}$ is stably finite at most one of the elements $x$ and $-x$ will belong to the positive cone in $K_{0}(\mathcal{B})$, [Bla1]. We know also (from $K_{1}(\mathcal{A})=0$ and Pimsner-Voiculescu's exact sequence) that at least one of the elements $x$ and $-x$ belongs to the image of the positive cone in $K_{0}(\mathcal{A})$ in $K_{0}(\mathcal{B})$. This shows that the positive cone in $K_{0}(\mathcal{B})$ is exactly the image of the positive cone in $K_{0}(\mathcal{A})$, which is identified with the non-negative dyadic numbers.

The $K$-theory of the inductive limit described in the beginning of this remark is given by:

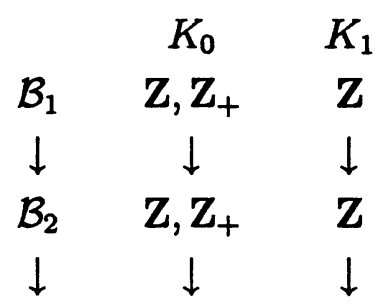

where all the vertical arrows on the $K$-groups are given by multiplication by 2 . Thus, if $\mathcal{C}$ is the inductive limit,

$$
K_{0}(\mathcal{C}) \cong \mathbf{Z}\left[\frac{1}{2}\right] \cong K_{1}(\mathcal{C})
$$

and the positive cone in $K_{0}(\mathcal{C})$ is the non-negative dyadic numbers. It follows that the ordered $K$-theory of $\mathcal{C}$ is isomorphic to that of $\mathcal{B}$, and hence $\mathcal{B} \cong \mathcal{C}$ by [Ell] or [Su].

\section{Voiculescu's almost inductive limit automorphisms}

If $\mathcal{A}$ is a unital $C^{*}$-algebra, $\mathcal{F}(\mathcal{A})$ denotes the set of finite dimensional *-subalgebras of $\mathcal{A}$ containing the unit of $\mathcal{A}$. If $\mathcal{B}, \mathcal{C}$ are subalgebras of $\mathcal{A}$ we will follow [Voi] in using the 
notation $\mathcal{B} \subset^{\varepsilon} \mathcal{C}$ if

$$
\sup \{\inf \{\|x-y\| \mid y \in \mathcal{C},\|y\| \leq 1\} \mid x \in \mathcal{B},\|x\| \leq 1\}<\varepsilon,
$$

and the distance $d(\mathcal{B}, \mathcal{C})$ between $\mathcal{B}$ and $\mathcal{C}$ is defined by

$$
d(\mathcal{B}, \mathcal{C})=\inf \left\{\varepsilon>0 \mid \mathcal{B} \subset^{\varepsilon} \mathcal{C} \text { and } \mathcal{C} \subset^{\varepsilon} \mathcal{B}\right\}
$$

Since any automorphism $\beta$ of the CAR algebra $\mathcal{A}$ is approximately inner it follows from [Voi, Lemma 3.1] that for any $\mathcal{D} \in \mathcal{F}(\mathcal{A})$ and any positive integer $m$, there are $\mathcal{B}_{j} \in \mathcal{F}(\mathcal{A})$, $j=0,1, \cdots, m$ with $\mathcal{B}_{0}=\mathcal{B}_{m}$ such that

$$
d\left(\beta\left(\mathcal{B}_{j}\right), \mathcal{B}_{j+1}\right)<\frac{5 \pi}{m}
$$

for $0 \leq j<m$, and

$$
\mathcal{D} \subseteq \mathcal{B}_{j}
$$

for $j=0,1, \cdots, m$. Thus, if $e_{i j}, i, j=0,1, \cdots, m-1$ is a complete set of matrix units for $M_{m}$, and $\sigma_{m}$ is the cyclic shift of $M_{m}$, defined through

$$
\sigma_{m}\left(e_{i j}\right)=e_{i+1, j+1}
$$

(where the addition is modulo $m$ ), and $\mathcal{E} \in \mathcal{F}\left(\mathcal{A} \otimes M_{m}\right)$ is defined through

$$
\mathcal{E}=\sum_{j=0}^{m-1} \mathcal{B}_{j} \otimes e_{j j}
$$

then

$\mathcal{D} \otimes 1 \subset \mathcal{E}$

and

$$
d\left(\left(\beta \otimes \sigma_{m}\right)(\mathcal{E}), \mathcal{E}\right)<\frac{5 \pi}{m} .
$$

Now suppose $\beta$ has the property that for any positive integer $m_{0}$ there is an integer $m>$ $m_{0}$ such that for every positive integer $N$ and every $\delta>0$ there is a subalgebra $\mathcal{C} \subset^{\delta}$ $\underset{k=N+1}{\otimes} M_{2} \subseteq M_{2^{\infty}}=\mathcal{A}$ such that $\mathcal{C}$ contains the unit of $\mathcal{A}, \mathcal{C} \cong M_{m}, \mathcal{C}$ has a cyclic shift $\sigma_{m}$, and $\left\|\beta(x)-\sigma_{m}(x)\right\| \leq \delta\|x\|$ for $x \in \mathcal{C}$. Then $\beta$ is an almost inductive limit automorphism of $\mathcal{A}$, i.e. for every $\mathcal{D} \in \mathcal{F}(\mathcal{A})$ and every $\varepsilon>0$ there is a $\mathcal{E} \in \mathcal{F}(\mathcal{A})$ such that $\mathcal{D} \subset^{\varepsilon} \mathcal{E}$ and $d(\beta(\mathcal{E}), \mathcal{E})<\varepsilon$ 
This is seen as follows: By modifying the $\mathcal{D}$ and $\mathcal{B}_{i}$ above by a small amount we may assume $\mathcal{B}_{i} \subseteq \underset{-N}{\otimes} M_{2}$ for some $N$, but then, integrating over the unitary group of ${\underset{-N}{\otimes}}_{N}^{N} M_{2}$ and

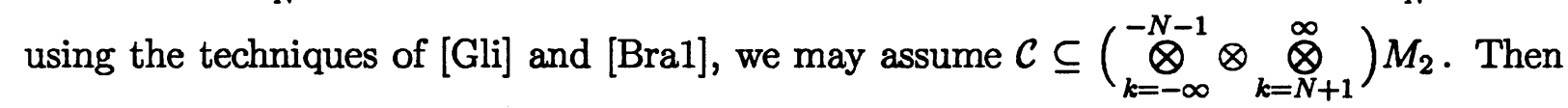
redefine the $\mathcal{E}$ above as $\mathcal{E}=\sum_{j=0}^{m-1} \mathcal{B}_{j} e_{j j}$. If $x=\sum_{j=0}^{m-1} b_{j} e_{j j} \in \mathcal{E}$ one computes

$$
\begin{aligned}
\left\|\beta(x)-" \beta \otimes \sigma_{m}^{\prime \prime}(x)\right\| & =\left\|\sum_{j=0}^{m-1} \beta\left(b_{j}\right)\left(\beta-\sigma_{m}\right)\left(e_{j j}\right)\right\| \\
& \leq \sum_{j=0}^{m-1}\left\|b_{j}\right\| \delta,
\end{aligned}
$$

where the notation " $\beta \otimes \sigma_{m}$ " is self explanatory. As $\|x\|=\sup _{j}\left\|b_{j}\right\|$, it follows that

$$
\left\|\beta(x)-^{\prime \prime} \beta \otimes \sigma^{\prime \prime}(x)\right\| \leq m \delta\|x\|
$$

Since we may choose this $\delta$ after $m$, we may make $\delta m$ as small as we want, and as

$$
d\left(\left(^{\prime \prime} \beta \otimes \sigma^{\prime \prime}\right)(\mathcal{E}), \mathcal{E}\right)<\frac{5 \pi}{m}
$$

we may make $d(\beta(\mathcal{E}), \mathcal{E})$ as small as desired.

Thus, it follows from [Voi, Proposition 2.3] that for any $\varepsilon>0$ there is a unitary $u \in \mathcal{A}$ such that $\|u-1\|<\varepsilon$, and $\gamma=A d u \circ \beta$ is an inductive limit automorphism, i.e. there exists an increasing sequence $\mathcal{A}_{n} \in \mathcal{F}(\mathcal{A})$ such that $\cup_{n} \mathcal{A}_{n}$ is dense in $\mathcal{A}$ and $\gamma\left(\mathcal{A}_{n}\right)=\mathcal{A}_{n}$. But then $\mathcal{A} \times{ }_{\gamma} \mathbf{Z}$ is the inductive limit of $\mathcal{A}_{n} \times{ }_{\gamma} \mathbf{Z}$, and each of these latter algebras has the form $\mathcal{D}_{n} \otimes C(\hat{\mathbf{Z}})=\mathcal{D}_{n} \otimes C(\mathbf{T})$ where $\mathcal{D}_{n}$ is the finite dimensional algebra obtained from $\mathcal{A}_{n}$ by merging the factors over the central orbits of $\gamma$ into one factor of dimension equal to the product of the dimensions of each factor by the order of the orbit [Bra 2]. Note also that $\mathcal{A} \times{ }_{\gamma} \mathbf{Z}$ is isomorphic to $\mathcal{A} \times{ }_{\beta} \mathbf{Z}$, [Tak]. In conclusion, we have the following known lemma:

Lemma 2.1 [Voi, Lemma 3.3] Let $\beta$ be an automorphism of $\mathcal{A}=M_{2 \infty}$ with the property that for any positive integer $m_{0}$ there is an integer $m>m_{0}$ such that for every positive integer $N$ and every $\varepsilon>0$ there is a subalgebra $\mathcal{C} \in \mathcal{F}(\mathcal{A})$ such that $\mathcal{C} \subset^{\varepsilon} \underset{k=N+1}{\infty} M_{2} \subseteq \mathcal{A}$, $\mathcal{C} \cong M_{m}$ and there exists a cyclic shift $\sigma_{m}$ of order $m$ on $\mathcal{C}$ such that

$$
\left\|\beta(x)-\sigma_{m}(x)\right\| \leq \varepsilon\|x\|
$$

for all $x \in \mathcal{C}$. 
It follows that $\beta$ is an almost inductive limit automorphism, and hence $\mathcal{A} \times{ }_{\beta} \mathbf{Z}$ contains an increasing sequence $\mathcal{B}_{n}$ of $C^{*}$-subalgebras such that $\cup_{n} \mathcal{B}_{n}$ is dense, and each $\mathcal{B}_{n}$ has the form

$$
\mathcal{B}_{n} \cong \bigoplus_{k=1}^{m_{n}} M_{[n, k]} \otimes C(\mathrm{~T})
$$

where $[n, k] \in \mathbf{N}$ and $m_{n}$ is finite.

\section{Quasifree automorphisms and the shift}

If $\mathcal{H}$ is a separable infinite-dimensional Hilbert space with inner product $($,$) , the algebra$ $\mathcal{A}=M_{2^{\infty}}$ can be described as the universal $C^{*}$-algebra generated by operators $a(f), f \in \mathcal{H}$, satisfying

$$
\begin{aligned}
& f \rightarrow a(f) \text { is antilinear, } \\
& a(f) a(g)+a(g) a(f)=0, \\
& a(f) a(g)^{*}+a(g)^{*} a(f)=(f, g) \mathbf{1},
\end{aligned}
$$

see e.g. [BR2, Theorem 5.2.5]. If $\left(f_{k}\right)_{k=1}^{\infty}$ is an orthonormal basis for $\mathcal{H}$, the matrix units $\left(e_{i j}^{(k)}\right)_{i, j=1}^{2}$ for the $k^{\prime}$ th tensor factor of $\mathcal{A}=\bigotimes_{k=1}^{\infty} M_{2}$ can be given by

$$
\begin{aligned}
e_{11}^{(k)}=a\left(f_{k}\right) a\left(f_{k}\right)^{*} & e_{12}^{(k)}=V_{k-1} a\left(f_{k}\right) \\
e_{21}^{(k)}=V_{k-1} a\left(f_{k}\right)^{*} & e_{22}^{(k)}=a\left(f_{k}\right)^{*} a\left(f_{k}\right),
\end{aligned}
$$

where

$$
\begin{aligned}
V_{k} & =\prod_{i=1}^{k}\left(1-2 a\left(f_{i}\right)^{*} a\left(f_{i}\right)\right) \\
& =\left(\begin{array}{cc}
1 & 0 \\
0 & -1
\end{array}\right) \otimes\left(\begin{array}{cc}
1 & 0 \\
0 & -1
\end{array}\right) \otimes \cdots \otimes\left(\begin{array}{cc}
1 & 0 \\
0 & -1
\end{array}\right) \otimes 1 \otimes 1 \otimes \cdots
\end{aligned}
$$

and there are $k$ factors $\left(\begin{array}{cc}1 & 0 \\ 0 & -1\end{array}\right)$. Conversely

$$
\begin{aligned}
a\left(f_{k}\right) & =\left(\prod_{l=1}^{k-1}\left(e_{11}^{(l)}-e_{22}^{(l)}\right)\right) e_{12}^{(k)} \\
& =\left(\begin{array}{cc}
1 & 0 \\
0 & -1
\end{array}\right) \otimes \cdots \otimes\left(\begin{array}{cc}
1 & 0 \\
0 & -1
\end{array}\right) \otimes\left(\begin{array}{ll}
0 & 1 \\
0 & 0
\end{array}\right) \otimes 1 \otimes 1 \otimes \cdots
\end{aligned}
$$


where there are $k-1$ factors $\left(\begin{array}{cc}1 & 0 \\ 0 & -1\end{array}\right)$.

If $V$ is any isometry on $\mathcal{H}, V$ defines a ${ }^{*}$-morphism of $\mathcal{A}$ by

$$
a(f) \rightarrow a(V f)
$$

(and this is a *automorphism if $V$ is unitary, called a quasi-free or Bogoliubov automorphism). In particular, let $\beta$ be the morphism defined by the one-sided shift:

$$
V f_{k}=f_{k+1}, \quad k=1,2, \ldots
$$

We call $\beta$ the one-sided quasi-free shift. On the other hand, let $\alpha$ be the usual one-sided shift on $\mathcal{A}$ :

$$
\alpha\left(e_{i j}^{(k)}\right)=e_{i j}^{(k+1)}, \quad k=1,2, \ldots
$$

i.e.

$$
\alpha(x)=\left(\begin{array}{ll}
1 & 0 \\
0 & 1
\end{array}\right) \otimes x
$$

Let $\gamma$ be the quasi-free automorphism defined by

$$
\gamma(a(f))=-a(f)
$$

i.e.

$$
\gamma\left(e_{i j}^{(k)}\right)=(-1)^{i-j} e_{i j}^{(k)}
$$

Define an element $x \in \mathcal{A}$ to be even if $\gamma(x)=x$ and odd if $\gamma(x)=-x$. Thus the ${ }^{*}$-algebra $\mathcal{A}^{e}$ of even elements is the closure of the set of polynomials in $a(f), a(f)^{*}$ with an even number of creators or annihilators in each constituent monomial. Using the expression of $a\left(f_{k}\right)$ in terms of the $e_{i j}^{(k)}$ 's, one now easily computes

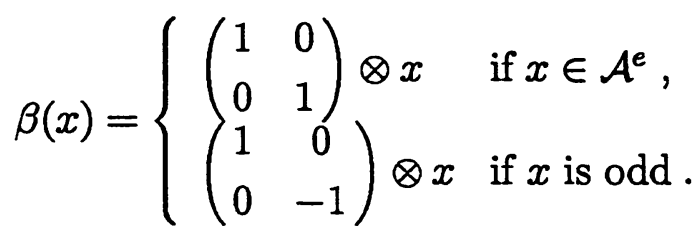

In particular we deduce

Lemma 3.1 The one-sided shift $\alpha$ and the one-sided quasifree shift $\beta$ have the same restrictions to the even algebra $\mathcal{A}^{e}$. 


\section{Almost shift-invariant matrix sub-algebras of $M_{2^{\infty}}$.}

Proposition 4.1 Let $\mathcal{A}=M_{2 \infty}=\bigotimes_{k=1}^{\infty} M_{2}$ and let $\alpha$ be the one-sided shift on $\mathcal{A}$. If $\varepsilon>0$ and $\rho_{1}, \rho_{2}, \cdots, \rho_{n} \in \mathbf{T} \subseteq \mathbf{C}$ then there exist a subalgebra $\mathcal{B} \in \mathcal{F}(\mathcal{A})$ such that $\mathcal{B} \cong M_{2^{n}}$, and an automorphism $\beta$ of $\mathcal{B}$ of the form

$$
\beta=A d\left(\left(\begin{array}{cc}
1 & 0 \\
0 & \rho_{1}
\end{array}\right) \otimes\left(\begin{array}{cc}
1 & 0 \\
0 & \rho_{2}
\end{array}\right) \otimes \cdots \otimes\left(\begin{array}{cc}
1 & 0 \\
0 & \rho_{n}
\end{array}\right)\right)
$$

relatively to some tensor product decomposition of $\mathcal{B}$, such that

$$
\|\alpha(x)-\beta(x)\| \leq \varepsilon\|x\|
$$

for all $x \in \mathcal{B}$.

Remark 4.2 Once this is true for the one-sided shift $\alpha$ it is also trivially true for the two-sided shift.

Remark 4.3 In particular, putting

$$
\rho_{k}=e^{2 \pi i 2^{-k}}, \quad k=1, \cdots, n,
$$

the spectrum of the unitary operator implementing $\beta$ becomes the set of all $2^{n}$-roots of 1 , and hence the proposition applies to the cyclic shift of order $2^{n}$ on $M_{2^{n}}$.

Proof of Proposition 4.1 First note that the two-sided shift $V$ on $L^{2}(\mathrm{Z})$ is a unitary operator with spectrum $\mathbf{T}$, and hence by spectral theory there exist for any $\delta>0$ mutually orthogonal unit vectors $\xi_{0}, \xi_{1}, \cdots, \xi_{n}$ in $L^{2}(\mathbf{Z})$ such that

$$
\begin{aligned}
& \left\|V \xi_{0}-\xi_{0}\right\|<\delta, \\
& \left\|V \xi_{k}-\rho_{k} \xi_{k}\right\|<\delta, \quad k=1, \cdots, n .
\end{aligned}
$$

Furthermore, shifting the $\xi_{k}$ 's sufficiently far to the right and changing each $\xi_{k}$ by a small amount we may assume

$$
\xi_{k} \in L^{2}(\mathbf{N}), \quad k=0,1, \cdots, n .
$$

Now, one checks that the operators $a_{k}$ defined by

$$
a_{k}=a\left(\xi_{k}\right)\left(a\left(\xi_{0}\right)+a\left(\xi_{0}\right)^{*}\right), \quad k=1, \cdots, n
$$


satisfy the anti-commutation relations

$$
\begin{aligned}
a_{k} a_{l}+a_{l} a_{k} & =0 \\
a_{k} a_{l}^{*}+a_{l}^{*} a_{k} & =\delta_{k, l} 1,
\end{aligned}
$$

so the ${ }^{*}$-algebra $B$ generated by the $a_{k}$ 's is isomorphic to $M_{2^{n}}$ by [BR2, Theorem 5.2.5]. Furthermore, the automorphism $\beta$ of $\mathcal{B}$ determined by

$$
\beta\left(a_{k}\right)=\bar{\rho}_{k} a_{k}, \quad k=1, \cdots, n,
$$

has the required form

$$
\beta=A d\left(\left(\begin{array}{cc}
1 & 0 \\
0 & \rho_{1}
\end{array}\right) \otimes \cdots \otimes\left(\left(\begin{array}{cc}
1 & 0 \\
0 & \rho_{n}
\end{array}\right)\right)\right.
$$

relatively to the matrix units defined by $a_{1}, \cdots, a_{n}$.

If $\sigma$ is the quasifree one-sided shift defined by $\left.V\right|_{L^{2}(\mathbf{N})}$, then

$$
\begin{aligned}
& \sigma\left(a_{k}\right)-\bar{\rho}_{k} a_{k} \\
& =a\left(V \xi_{k}\right)\left(a\left(V \xi_{0}\right)+a\left(V \xi_{0}\right)^{*}\right)-a\left(\rho_{k} \xi_{k}\right)\left(a\left(\xi_{0}\right)+a\left(\xi_{0}\right)^{*}\right) .
\end{aligned}
$$

Thus, using $\|a(\xi)\|=\|\xi\|$ we deduce

$$
\begin{aligned}
& \left\|\sigma\left(a_{k}\right)-\bar{\rho}_{k} a_{k}\right\| \\
& \quad \leq 2\left\|V \xi_{k}-\rho_{k} \xi_{k}\right\|+2\left\|V \xi_{0}-\xi_{0}\right\| \\
& \quad<4 \delta .
\end{aligned}
$$

Since any element in $\mathcal{B}$ is a polynomial in the $a_{k}, a_{k}^{*}$ of degree at most $n$, it follows by choosing $\delta$ small enough that

$$
\|\sigma(x)-\beta(x)\| \leq \varepsilon\|x\|
$$

for all $x \in \mathcal{B}$. Finally, as the $a_{k}$ are polynomials of homogeneous degree 2 in creators and annihilators, $\mathcal{B} \subseteq \mathcal{A}^{e}$ and it follows from Lemma 3.1 that $\left.\alpha\right|_{\mathcal{B}}=\left.\sigma\right|_{\mathcal{B}}$. Thus Proposition 4.1 follows. 


\section{The shift is an almost inductive limit automor- phism}

The proof of Theorem 1.1 is now immediate: By Remark 4.2 there exist for any $\varepsilon>0$ and any $n$ a subalgebra $\mathcal{B}$ of $\mathcal{A}$ containing the unit of $\mathcal{A}$ such that $\mathcal{B} \cong M_{2^{n}}$ and an automorphism $\beta$ of $\mathcal{B}$ isomorphic to the cyclic shift of order $2^{n}$ such that

$$
\|\alpha(x)-\beta(x)\| \leq \varepsilon\|x\|
$$

for all $x \in \mathcal{B}$. Since the latter estimate is not changed by the replacement $\mathcal{B} \rightarrow \alpha^{m}(\mathcal{B})$, $\beta \rightarrow \alpha^{m} \beta \alpha^{-m}$, we may also assume that $\mathcal{B} \subset^{\varepsilon} \bigotimes_{k=N+1}^{\infty} M_{2}$ for any given $N$. Theorem 1.1 now follows from Lemma 2.1 .

Remark 5.1 By pushing the ideas from [Voi] and using the techniques in [BKR, Proposition 2.12] one could prove Theorem 1.1 if one could establish that for any $\varepsilon>0$ there is a unitary $u \in \mathcal{A}$ such that $\|u-1\|<\varepsilon$, and the $C^{*}$-dynamical system $(\mathcal{A}, A d u \circ \alpha)$ is isomorphic to $(\mathcal{A} \otimes \mathcal{A}, \beta \otimes \sigma)$, where $\sigma=\bigotimes_{n=1}^{\infty} \sigma_{2^{n}}$ on $\mathcal{A} \cong \bigotimes_{n=1}^{\infty} M_{2^{n}}$, and $\sigma_{2^{n}}$ is the cyclic shift of order $2^{n}$ on $M_{2^{n}}$, and $\beta$ some automorphism of $\mathcal{A}$. One may then appeal directly to [Voi, Lemma 3.3] to prove that $A d u \circ \alpha$ is an almost inductive limit automorphism.

\section{Divisibility of $M_{2^{\infty}} \times_{\alpha} \mathrm{Z}$}

In this section we will prove Corollary 1.2, i.e.

$$
\mathcal{B}=M_{2 \infty} \times_{\alpha} \mathbf{Z} \cong\left(M_{2 \infty} \times_{\alpha} \mathbf{Z}\right) \otimes M_{2 \infty}
$$

To this end we will combine Proposition 4.1 with a special case of a result in [BKR]:

Lemma 6.1 [BKR, Proposition 2.12] Let $\mathcal{A}$ be a unital separable $C^{*}$-algebra with the property that for any finite set $\left\{x_{1}, \cdots, x_{n}\right\} \in \mathcal{A}$ and any $\varepsilon>0$ there exists a $\mathcal{B} \in \mathcal{F}(\mathcal{A})$ such that $\mathcal{B} \cong M_{2}$ and $\left\|\left[x_{i}, y\right]\right\| \leq \varepsilon\|y\|$ for $i=1,2, \cdots, n$ and all $y \in \mathcal{B}$. It follows that

$$
\mathcal{A} \cong \mathcal{A} \otimes M_{2 \infty}
$$


Proof: By [BKR, Proposition 2.12 and its proof], $\mathcal{A} \cong \mathcal{C} \otimes M_{2 \infty}$ for a suitable $C^{*}$-algebra C. But as $M_{2 \infty} \otimes M_{2 \infty} \cong M_{2 \infty}$ it follows that

$$
\begin{aligned}
\mathcal{A} & \cong \mathcal{C} \otimes\left(M_{2^{\infty}} \otimes M_{2 \infty}\right) \\
& \cong\left(\mathcal{C} \otimes M_{2^{\infty}}\right) \otimes M_{2^{\infty}} \\
& \cong \mathcal{A} \otimes M_{2^{\infty}} .
\end{aligned}
$$

To prove Corollary 1.2 , note that, by Proposition 4.1 , for any $\varepsilon>0$ there is a $\mathcal{C} \in \mathcal{F}(\mathcal{A})$ such that $\mathcal{C} \cong M_{2}$ and $\|\alpha(x)-x\| \leq \varepsilon\|x\|$ for all $x \in \mathcal{C}$. Replacing $\mathcal{C}$ by $\alpha^{m}(\mathcal{C})$ we may assume that $\mathcal{C}$ approximately commutes with any finite subset of $M_{2 \infty}$, and as this replacement does not affect the estimate $\|\alpha(x)-x\| \leq \varepsilon\|x\|$ the new $\mathcal{C}$ (as well as the old) approximately commutes with the canonical unitary $u$ in the crossed product $\mathcal{B}=M_{2^{\infty}} \times_{\alpha} \mathbf{Z}$.

Corollary 1.2 now follows from Lemma 6.1.

\section{The Cuntz algebra $\mathrm{O}_{2}$}

Recall from [Cun] that $\mathcal{O}_{2}$ is the universal $C^{*}$-algebra generated by two operators $S_{1}, S_{2}$ satisfying the relations

$$
1=S_{1}^{*} S_{1}=S_{2}^{*} S_{2}=S_{1} S_{1}^{*}+S_{2} S_{2}^{*}
$$

We will prove Corollary 1.3 , i.e.

$$
\mathcal{O}_{2} \cong \mathcal{O}_{2} \otimes M_{2 \infty}
$$

Recall from [Cun] that $\mathcal{O}_{2}$ contains $M_{2}$ canonically as a unital sub-algebra as follows: If $\mu$ is a multiindex of length $n$ with values in $\{1,2\}$, i.e. $\mu=\left(\mu_{1}, \cdots, \mu_{n}\right)$ with $\mu_{j} \in\{1,2\}$, define $S_{\mu}=S_{\mu_{1}} S_{\mu_{2}} \cdots S_{\mu_{n}}$. Then the set of

$$
S_{\mu} S_{\nu}^{*}
$$

where $\mu, \nu$ run over the multiindices of length $n$, constitute a complete set of $2^{n} \times 2^{n}$ matrix units. Letting $n \rightarrow \infty$, one establishes that the fixed point subalgebra $\mathcal{A}$ of $\mathcal{O}_{2}$ under the gauge group $\rho \in \mathbf{T} \rightarrow \sigma_{\rho}$, where $\sigma_{\rho}\left(S_{1}\right)=\rho S_{1}, \sigma_{\rho}\left(S_{2}\right)=\rho S_{2}$, is isomorphic to $M_{2^{\infty}}$.

Now, define a morphism $\phi$ of $\mathcal{O}_{2}$ by

$$
\phi(x)=S_{1} x S_{1}^{*}+S_{2} x S_{2}^{*}
$$

Then $\phi$ commutes with the gauge action, so $\phi(\mathcal{A}) \subseteq \mathcal{A}$, and by applying $\phi$ to the matrix units $S_{\mu} S_{\nu}^{*}$, one sees that the restriction of $\phi$ to $\mathcal{A}$ is the one-sided shift. It follows from 
Proposition 4.1 that for any $\varepsilon>0$ there is a ${ }^{*}$ subalgebra $\mathcal{B}$ of $\mathcal{A}$ containing the unit of $\mathcal{A}$ such that $\mathcal{B} \cong M_{2}$ and such that

$$
\|\phi(x)-x\| \leq \varepsilon\|x\|
$$

for all $x \in \mathcal{B}$. But

$$
\begin{aligned}
(\phi(x)-x) S_{1} & =S_{1} x S_{1}^{*} S_{1}+S_{2} x S_{2}^{*} S_{1}-x S_{1} \\
& =S_{1} x-x S_{1}=\left[S_{1}, x\right]
\end{aligned}
$$

and correspondingly

$$
\begin{aligned}
& S_{1}^{*}(\phi(x)-x)=\left[x, S_{1}^{*}\right], \\
& (\phi(x)-x) S_{2}=\left[S_{1}, x\right], \\
& S_{2}(\phi(x)-x)=\left[x, S_{2}^{*}\right],
\end{aligned}
$$

so the commutator of $x \in \mathcal{B}$ with any monomial in $S_{i}, S_{i}^{*}$ of order 1 has norm less than or equal to $\varepsilon\|x\|$. Since the polynomials in $S_{i}, S_{i}^{*}$ are dense in $\mathcal{O}_{2}$ it follows that $\mathcal{O}_{2}$ is approximately divisible in the sense that for all finite sequences $x_{1}, \cdots, x_{n} \in \mathcal{O}_{2}$ and all $\varepsilon>0$, there exists a ${ }^{*}$-subalgebra $\mathcal{B} \subseteq \mathcal{O}_{2}$ containing the unit of $\mathcal{O}_{2}$ such that

$$
\left\|\left[x_{i}, x\right]\right\| \leq \varepsilon\|x\|
$$

for all $x \in \mathcal{B}$, and $\mathcal{B} \cong M_{2}$. It therefore follows from Lemma 6.1 that

$$
\mathcal{O}_{2} \cong \mathcal{O}_{2} \otimes M_{2 \infty}
$$

\section{Complements on almost shift-invariant projections}

Let $\alpha$ be the shift on $M_{2^{\infty}}$. It follows from Proposition 4.1 that for any $\varepsilon>0$ we may find an $m$ and a nontrivial projection

$$
E \in \bigotimes_{1}^{m} M_{2} \subseteq \bigotimes_{-\infty}^{\infty} M_{2}
$$

such that $\|\alpha(E)-E\|<\varepsilon$. The following proposition says that $m \geq \pi / 2 \arcsin \varepsilon$. 
Proposition 8.1 If $E \in \underset{1}{\otimes} M_{2}$ is a nontrivial projection, then

$\left\|E \otimes 1_{2}-1_{2} \otimes E\right\| \geq \sin (\pi / 2 m)$.

When $m=2$, this is the best possible estimate. (Here $E \otimes 1_{2}$ and $1_{2} \otimes E$ are both viewed as projections in $\bigotimes_{1}^{m+1} M_{2}$ ).

Proof: View $\bigotimes_{1}^{m} M_{2}$ as a subalgebra of $\bigotimes_{-\infty}^{\infty} M_{2}=M_{2^{\infty}}$, let $\tau$ be the trace state on $M_{2^{\infty}}$, and let $\mathcal{H}$ be the Hilbert space of the cyclic representation associated with $\tau$. Since $\tau$ is invariant under the shift $\alpha$, there is a unitary operator $U$ on $\mathcal{H}$ such that

$$
\alpha(x)=U x U^{*}
$$

for all $x \in M_{2^{\infty}}$. We have the identification

$$
1_{2} \otimes E=\alpha\left(E \otimes 1_{2}\right)=\alpha(E)=U E U^{*}
$$

so we must show

$$
\left\|E-U E U^{*}\right\| \geq \sin (\pi / 2 m)
$$

But

$$
U^{m} E U^{-m}=\alpha_{m}(E) \in \bigotimes_{m+1}^{2 m} M_{2}
$$

thus $E$ and $U^{m} E U^{-m}$ are distinct commuting projections, and there exists a nonzero vector $\xi \in \mathcal{H}$ such that

$$
E \xi=\xi \quad, U^{m} E U^{-m} \xi=0
$$

i.e. $\xi \in E \mathcal{H}$ and $U^{-m} \xi \perp E \mathcal{H}$. Since the angle between $U^{-m} \xi$ and $E \mathcal{H}$ is $\pi / 2$ it follows by weak compactness of the unit ball in $\mathcal{H}$ that there is another unit vector $\xi \in E \mathcal{H}$ such that the angle between $U^{-1} \xi$ and $E \mathcal{H}$ is at least $\pi / 2 m$ (since the maximum such angle between $U^{-k} E \mathcal{H}$ and $U^{-1} \xi$ over all $\xi \in U^{-k} E \mathcal{H}$ is the same for $k=0,1, \cdots, m-1$, and the sum of these angles is at least $\pi / 2$ ). But this means that

$$
\left\|E U^{-1} \xi\right\| \leq \cos (\pi / 2 m)
$$


and then

$$
\left\|E U^{*} \xi-U^{*} \xi\right\| \geq \sin (\pi / 2 m)
$$

i.e.

$$
\left\|U E U^{*} \xi-\xi\right\| \geq \sin (\pi / 2 m)
$$

and thus

$$
\|\alpha(E)-E\| \geq \sin (\pi / 2 m)
$$

Let us now look at the case $m=2$, where the lower bound becomes

$$
\left\|E \otimes 1_{2}-1_{2} \otimes E\right\| \geq 1 / \sqrt{2} \text {. }
$$

This lower bound is actually achieved by the projection

$$
E=\left(\begin{array}{cccc}
\frac{1}{2} & \frac{1}{2} & 0 & 0 \\
\frac{1}{2} & \frac{1}{2} & 0 & 0 \\
0 & 0 & \frac{1}{2} & -\frac{1}{2} \\
0 & 0 & -\frac{1}{2} & \frac{1}{2}
\end{array}\right)
$$

as one may verify by direct computation, or by noting that $E$ is a solution of the braid relation:

$$
\left(E \otimes 1_{2}\right)\left(1_{2} \otimes E\right)\left(E \otimes 1_{2}\right)=\frac{1}{2}\left(E \otimes 1_{2}\right)
$$

\section{References}

[BBEK] B. Blackadar, O. Bratteli, G. A. Elliott and A. Kumjian, Reduction of real rank in inductive limits of $C^{*}$-algebras, Math. Ann. 292 (1992), 111-126.

[BDR] B. Blackadar, M. Dadarlat, M. Rørdam, The real rank of inductive limit $C^{*}$ algebras, Math. Scand. 69 (1991), 211-216.

[Bed] E. Bedos, On the uniqueness of the trace on some simple $C^{*}$-algebras, preprint 1991.

[BRK] B. Blackadar, A. Kumjian and M. Rørdam, Approximately central matrix units and the structure of noncommutative tori, preprint 1991.

[Bla1] B. Blackadar, K-Theory for Operator Algebras, MSRIP 5, Springer Verlag 1986. 
[Bla2] B. Blackadar, Symmetries of the CAR algebra, Ann. of Math. (2) 131 (1990), 589623.

[BP] L. G. Brown and G. K. Pedersen, $C^{*}$-algebras of real rank zero, J. Functional Anal. 99 (1991), 131-149.

[BR1] O. Bratteli and D. W. Robinson, Operator Algebras and Quantum Statistical Mechanics I, Second Edition, Springer Verlag (1987).

[BR2] O. Bratteli and D. W. Robinson, Operator Algebras and Quantum Statistical Mechanics II, Springer Verlag (1981).

[Bra1] O. Bratteli, Inductive limits of finite dimensional $C^{*}$-algebras, Trans. Amer. Math. Soc. 171 (1972), 195-234.

[Bra2] O. Bratteli, Crossed product of UHF algebras by product type actions, Duke Math. J. 46 (1979), 1-23.

[Cun] J. Cuntz, Simple $C^{*}$-algebras generated by isometries, Commun. Math. Phys. 57 (1977), 173-185.

[Ell] G. A. Elliott, On the classification of $C^{*}$-algebras of real rank zero, preprint.

[Gli] J. Glimm, On a certain class of operator algebras, Trans. Amer. Math. Soc. 95 (1960), $318-340$.

[Kis] A. Kishimoto, Outer automorphisms and reduced crossed products of simple $C^{*}$ algebras, Commun. Math. Phys. 81 (1981), 429-435.

[Su] H. Su, On the classification of $C^{*}$-algebras of real rank zero: Inductive limits of matrix algebras over non-Hausdorff graphs, preprint 1992.

[Tak] M. Takesaki, Covariant representations of $C^{*}$-algebras and their locally compact automorphism groups, Acta Math. 119 (1967), 273-303.

[Voi] D. Voiculescu, Almost inductive limit automorphisms and embeddings into AFalgebras, Ergod. Th. \& Dynam. Sys. 6 (1986), 475-484. 